\title{
Integrative analysis of DNA copy number and gene expression in metastatic oral squamous cell carcinoma identifies genes associated with poor survival
}

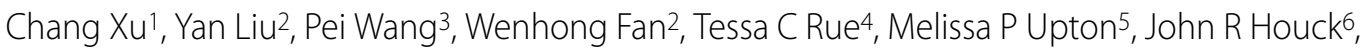 \\ Pawadee Lohavanichbutr6, David R Doody6, Neal D Futran', Lue Ping Zhao 2,4, Stephen M Schwartz ${ }^{6,7}$, Chu Chen 1,6,7 \\ and Eduardo Méndez $z^{* 1,6,8,9}$
}

\begin{abstract}
Background: Lymphotropism in oral squamous cell carcinoma (OSCC) is one of the most important prognostic factors of 5-year survival. In an effort to identify genes that may be responsible for the initiation of OSCC lymphotropism, we examined DNA copy number gains and losses and corresponding gene expression changes from tumor cells in metastatic lymph nodes of patients with OSCC.

Results: We performed integrative analysis of DNA copy number alterations (CNA) and corresponding mRNA expression from OSCC cells isolated from metastatic lymph nodes of 20 patients using Affymetrix $250 \mathrm{~K}$ Nsp I SNP and U133 Plus 2.0 arrays, respectively. Overall, genome CNA accounted for expression changes in 31\% of the transcripts studied. Genome region 11q13.2-11q13.3 shows the highest correlation between DNA CNA and expression. With a false discovery rate $<1 \%, 530$ transcripts (461 genes) demonstrated a correlation between CNA and expression. Among these, we found two subsets that were significantly associated with OSCC $(n=122)$ when compared to controls, and with survival $(n=27)$, as tested using an independent dataset with genome-wide expression profiles for 148 primary OSCC and 45 normal oral mucosa. We fit Cox models to calculate a principal component analysis-derived risk-score for these two gene sets ('122-' or '27-transcript PC'). The models combining the 122- or 27-transcript PC with stage outperformed the model using stage alone in terms of the Area Under the Curve (AUC $=0.82$ or 0.86 vs. 0.72 , with $p=0.044$ or 0.011 , respectively).

Conclusions: Genes exhibiting CNA-correlated expression may have biological impact on carcinogenesis and cancer progression in OSCC. Determination of copy number-associated transcripts associated with clinical outcomes in tumor cells with an aggressive phenotype (i.e., cells metastasized to the lymph nodes) can help prioritize candidate transcripts from high-throughput data for further studies.
\end{abstract}

\section{Background}

Oral squamous cell carcinoma (OSCC) is the sixth most common cancer worldwide. The presence of lymph node metastasis is associated with a $50 \%$ decrease in 5 -yr survival, and is the single most important prognostic factor identified to date [1-4]. However, the mechanisms by

* Correspondence: edmendez@u.washington.edu

1 Department of Otolaryngology-Head and Neck Surgery, University of Washington, Seattle, WA 98195, USA

Full list of author information is available at the end of the article which OSCC cells spread from the primary site to local lymph nodes is not well understood. Transcriptome profiling has been used to gain insights into this process [1,57], but the function of many of the proposed differentially expressed transcripts is unknown. To improve the likelihood of finding genes driving the carcinogenic process, several groups have exploited the common feature of genomic instability in cancer [8] and identified genes the expression of which is correlated with corresponding

() 2010 Xu et al; licensee BioMed Central Ltd. This is an Open Access article distributed under the terms of the Creative Commons Attri:HW 1 ed Central bution License (http://creativecommons.org/licenses/by/2.0), which permits unrestricted use, distribution, and reproduction in any medium, provided the original work is properly cited. 
DNA copy number in tumors such as brain, breast, ovarian, liver, multiple myeloma, and melanoma [9-18].

In an attempt to identify novel driver genes responsible for the OSCC metastasis, we utilized a recently developed protocol by our group for high-throughput profiling of DNA and RNA from the same cell population obtained by laser capture microdissection (LCM) to determine the association between DNA copy number aberration (CNA) and gene expression in tumor cells isolated from metastatic lymph nodes. We reasoned that these cells would contain those changes in the genome and transcriptome that are essential to the lymphotropism of OSCC. In addition, we tested the hypothesis that since nodal metastases are associated with poor prognosis, the expression of copy number-associated genes from metastatic OSCC tumor cells is associated with survival.

\section{Results}

\section{Study population}

Selected characteristics of the 20 OSCC patients with lymph node metastases are shown in Additional file 1, Table S1. Eight patients had cancers arising in the oropharynx whereas the remainder of the tumors arose from the oral cavity. The age range was 23-84 (mean 56.8) years. With the exception of three patients with only one positive lymph node, the majority of patients had $\geq \mathrm{N} 2$ nodal staging (i.e. multiple metastatic nodes detected).

\section{DNA copy number aberrations in OSCC nodal metastasis}

CNA events were detected in all of the 20 OSCC lymph node metastases and in all chromosomal arms that were covered with SNP probes (13p, 14p, 15p, 21p and 22p were not covered by the Affymetrix $250 \mathrm{~K}$ Nsp SNP array). The percentage of each genome showing CNA ranged between $25.6 \%-73.9 \%$ (mean $\pm \mathrm{sd}$ : $48.7 \pm 13.6 \%$. CNA was defined as the ratio of DNA copy number in cancer cells vs. normal cells either $<0.93$ or $>1.07$. In particular, large regions of amplification were detected on chromosome arms 3q, 5p, 8q, and 9q and large regions of deletion were detected on chromosome arms 3p, 5q, 8p, and 13q. Most of the previously reported CNA that have previously been associated with poor outcome in patients with OSCC or head and neck squamous cell carcinoma [19] were detected in the samples, such as amplification in regions 3q21-29, 5p15, 7p12, 8q21-24, 11q13, 12q24, $14 \mathrm{q} 23-32,16 \mathrm{q} 22$ and $20 \mathrm{q}$, and deletion in regions $1 \mathrm{p} 21$, 5q11-12, 5q14-15, 5q31, 8p21-22, 9p21, 10p12, 11q23-25, $12 q 22,18 q 11.2,21 q$ and $22 q$ (see Additional file 2, Figure S1).

\section{Inferring genome DNA copy number for each transcript} Out of the 54,675 transcripts measured by the Affymetrix U133 Plus 2.0 array, 23,484 transcripts (43.0\%) remained after filtering as described in the Methods. However, the majority of these transcripts did not overlap with a SNP that could be used for inferring the DNA copy number (Additional file 3, Table S2). Therefore, we incorporated the SNPs within a $250 \mathrm{~kb}$ upstream and downstream of neighboring region for each transcript. For 18 transcripts there were no SNPs within the $250 \mathrm{~kb}$ neighboring region, leaving 23,466 transcripts for determining the relationship between DNA copy number and gene expression. Out of these, the DNA copy number was inferred from at least 5 SNPs for 23,319 transcripts (99.4\% of the 23,466 transcripts) and from 1-4 SNPs, for 147 transcripts (Additional file 3, Table S2).

\section{Association of DNA copy number on gene expression}

Based on the distribution of the cancer-normal DNA copy number ratio, the 23,466 transcripts were divided into six subgroups, representing high level deletion (ratio $<0.5$ ), low level deletion (ratio: 0.5-0.93), no change (ratio: 0.93-1.07), low- (ratio: 1.07-2), medium- (ratio: 24 ), and high-level amplification (ratio > 4), respectively. The average gene expression in the low level genome amplification and deletion subgroups differed little from that in the no change subgroup (Figure 1A). However, the average gene expression in the high level deletion subgroup was -0.8 (base-2 log-transformed, which is equivalent to 1.7-fold lower) and the average gene expression in the medium and high level amplification subgroups was 0.7 and 1.6 (equivalent to 1.6-fold and 3.0-fold higher), respectively, as compared to that in the no change subgroup (Figure 1A). Thus, there was a dose-response relationship between DNA copy number and gene expression (Figure 1A).

To determine the relationship between DNA copy number and individual gene expression, we applied a robust linear regression model. The distribution of the correlation coefficients forms a normal-shaped curve with the mean of the distribution shifted to the positive direction (Figure 1B), indicating a large set of the genes having positive correlations between DNA copy number and gene expression. Based on the p-value distribution, we estimated that $30.6 \%$ of the transcripts showed statistically significant correlations between DNA copy number and expression (Figure 1C). With a False Discovery Rate $($ FDR) < 1\%, we identified 530 transcripts (461 genes) with a significantly correlated DNA copy number and expression.

\section{Genome region 11q13.2-q13.3 shows the highest correlation between DNA CNA and expression in metastatic OSCC}

Although we observed significant associations between gene expression and CNA on all chromosomal arms covered with SNP probes (Figure 2A), chromosomal region 11q13.2-q13.3 contained a cluster of genes with signifi- 


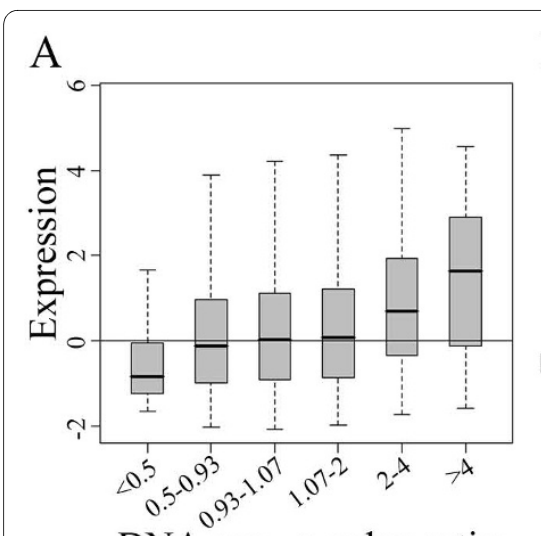

DNA copy number ratio

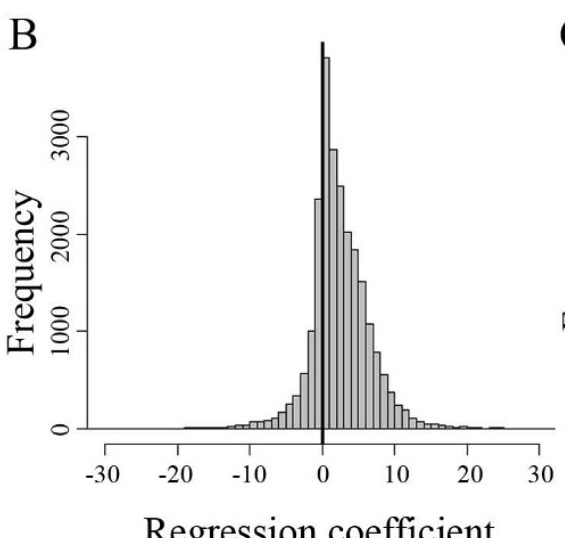

Regression coefficient

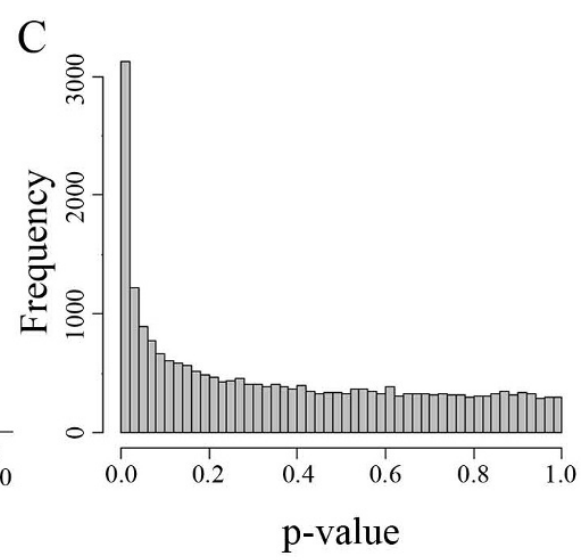

Figure 1 Influence of DNA copy number on gene expression in the $\mathbf{2 0}$ lymph node metastatic OSCC. A. Summary of gene expression in subgroup of transcripts with different cancer-normal DNA copy number ratio (Box plot in each subgroup indicates $25^{\text {th }}, 50^{\text {th }}$, and $75^{\text {th }}$ percentile of the gene expression). X-axis: subgroups with different DNA copy number ratio. Y-axis: base-2 log-transformed relative gene expression; B. Distribution of the estimated correlation coefficients between DNA copy number and gene expression. X-axis: value of the correlation coefficient; Y-axis: frequency; C. P-value distribution for the correlation coefficients.

cantly high correlation between DNA copy number and gene expression (i.e. $p<4.26 \times 10-7$, Bonferroni correction value) (Figure 2B). We further examined the genes in the region as previous studies had associated DNA amplification in this region with poor prognosis in OSCC patients [20]. There were 20 probe sets in this region that measured the expression of 12 genes (Figure 3A). Seven of these genes, CPT1A, MRPL21, TPCN2, ORAOV1, $F A D D$, and PPFIA1, and an unknown transcript measured by the probe set 236113_at, showed high correlation between DNA copy number and gene expression. The remaining five genes in the same region, IGHMBP2, MRGPRF, MYEOV, CCND1, and ANO1, showed no significant correlation between DNA copy number and gene expression (Figure 3B).

\section{Copy number-associated transcripts can distinguish OSCC} from normal oral mucosa and are associated with survival

To prioritize the 530 transcripts whose CNA and expression are significantly correlated, we examined their relationship with OSCC (vs. normal oral mucosa from control individuals) and with survival. We used a previously reported dataset containing transcriptome profiles for 167 OSCC primary tumors and 45 controls [21]. The baseline characteristics of the OSCC patients included in the survival analysis were presented previously in Méndez et al (Additional file 1, Table S1) [22]. Nineteen of the 20 patients who contributed lymph nodes for this study were also part of the 167 patients in the testing dataset. They were therefore excluded from further analysis. From the remaining 148 cases, 132 had at least 4 months of follow-up, a cut-off used to reduce the inclusion of deaths from patients due to co-morbidities rather than tumor biology. The range of follow-up time for these patients known to be alive from initial enrollment (December 16, 2003) to the date of last follow-up (April 17,2007 ) was 4.4 to 38.7 months, with a median of 17.0 months. Thirty six patients had died by the end of the follow-up period; there were 25 OSCC-specific deaths, nine non-OSCC-specific deaths and two deaths of unknown causes. To test the hypothesis that these 530 transcripts were associated with survival, we estimated hazard ratios (HR) for OSCC-specific mortality, adjusting for age and sex. Although the expression profiles in this dataset were generated using primary tumors and the DNA copy number-associated transcripts identified in this study was based on lymph node metastases, we hypothesized some of the changes have already happened in the primary tumors. The selected 530 transcripts were significantly enriched with genes differentially expressed in OSCC cases versus controls $(p<0.001)$ when compared with random gene sets of the same size. The list was also significantly enriched with genes associated with survival ( $p$ $<0.001)$. With the same cut-off value of Z-score $>6$ as in our previous study [21], 122 transcripts representing 116 genes were identified as differentially expressed in OSCC cases versus controls (Additional file 4, Table S3). Ingenuity Pathways Analysis (IPA v8.5, Ingenuity ${ }^{\circ}$ Systems, http:/ /www.ingenuity.com was able to functionally categorize 88 of the 116 genes, of which the following molecular and cellular functional categories with the largest number of genes involved: "Gene Expression" (25 genes: ACTL6A, ATF7, BAG1, CCND2, EIF2S1, EN1, ERCC5, FADD, GTF2E1, HLTF, MBD2, MCM7, MUC1, MYNN, NFX1, OSMR, PAK2, PBX1, RAF1, RBBP8, SLU7, SNAPC3, TAF6, VAV3, and ZHX1), "Cell Cycle" (15 genes: 


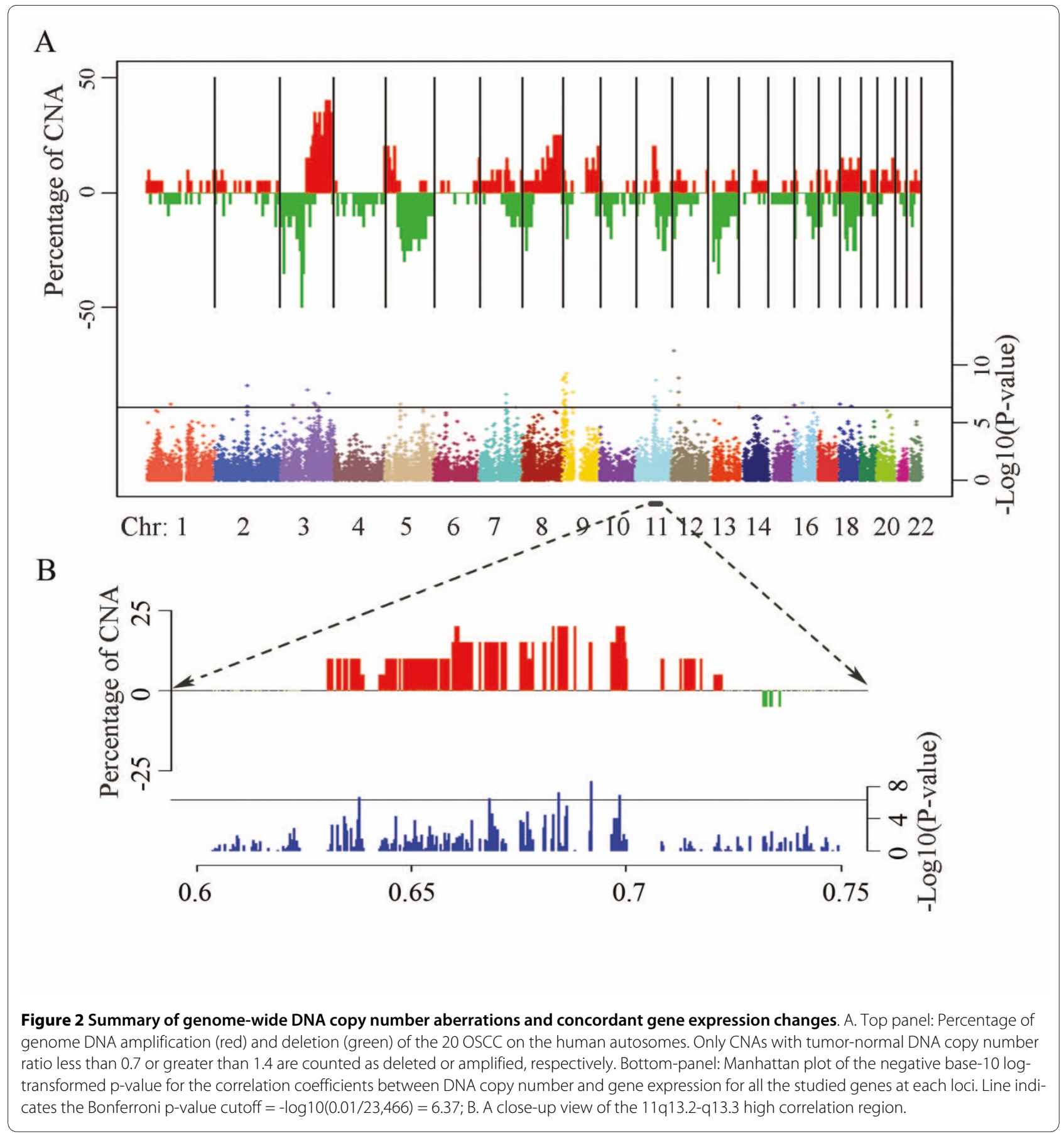

ACTL6A, CCND2, CSNK2A2, FADD, MCM7, MPHOSPH6, PAK2, PCM1, PPP2R2A, RAF1, RBBP8, SENP5 (includes EG:205564), SSSCA1, TAF6, and VAV3), and "Cell Death" (12 genes: ATF7, BAG1, CCND2, CSNK2A2, EN1, FADD, MUC1, PAK2, PBX1, PPP2R2A, $R A F 1$, and $T A F 6$ ). In addition, 27 transcripts representing 24 genes were associated with survival (Table 1). IPA was able to functionally categorize 19 of the 24 genes, of which the following molecular and cellular functional categories with the largest number of genes involved:
"Cell Death" (CCT6A, FADD, NFE2L2, RAD23B, STEAP3, and TREM1) and "Cellular Movement" (FADD, NFE2L2, and PPFIA1).

Given results from our previous study showing that genes that are differentially expressed between OSCC and normal oral mucosa are associated with OSCC prognosis [22], we tested whether gene expression levels of the 122 transcripts were associated with survival. We used the first two principal component (PC) scores of the 122 transcripts as a predictor to fit Cox models. We also 
A

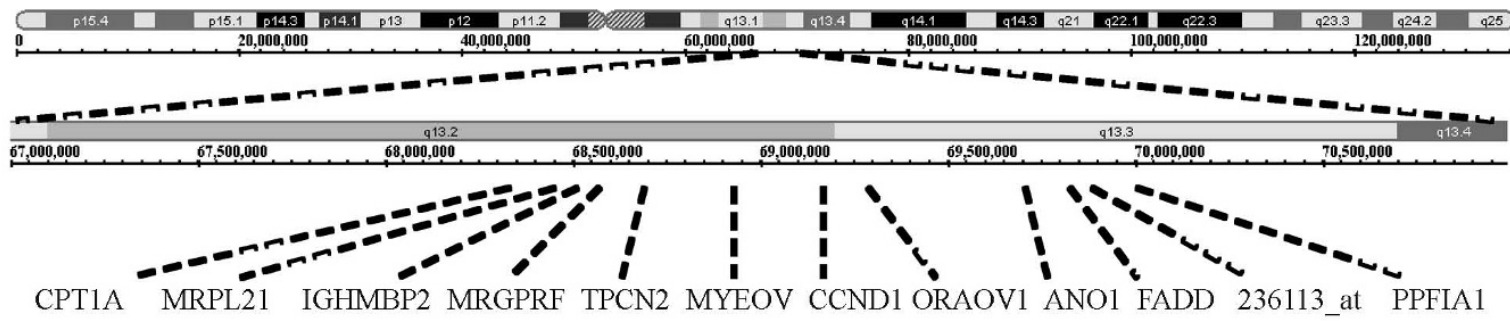

$\mathrm{B}$
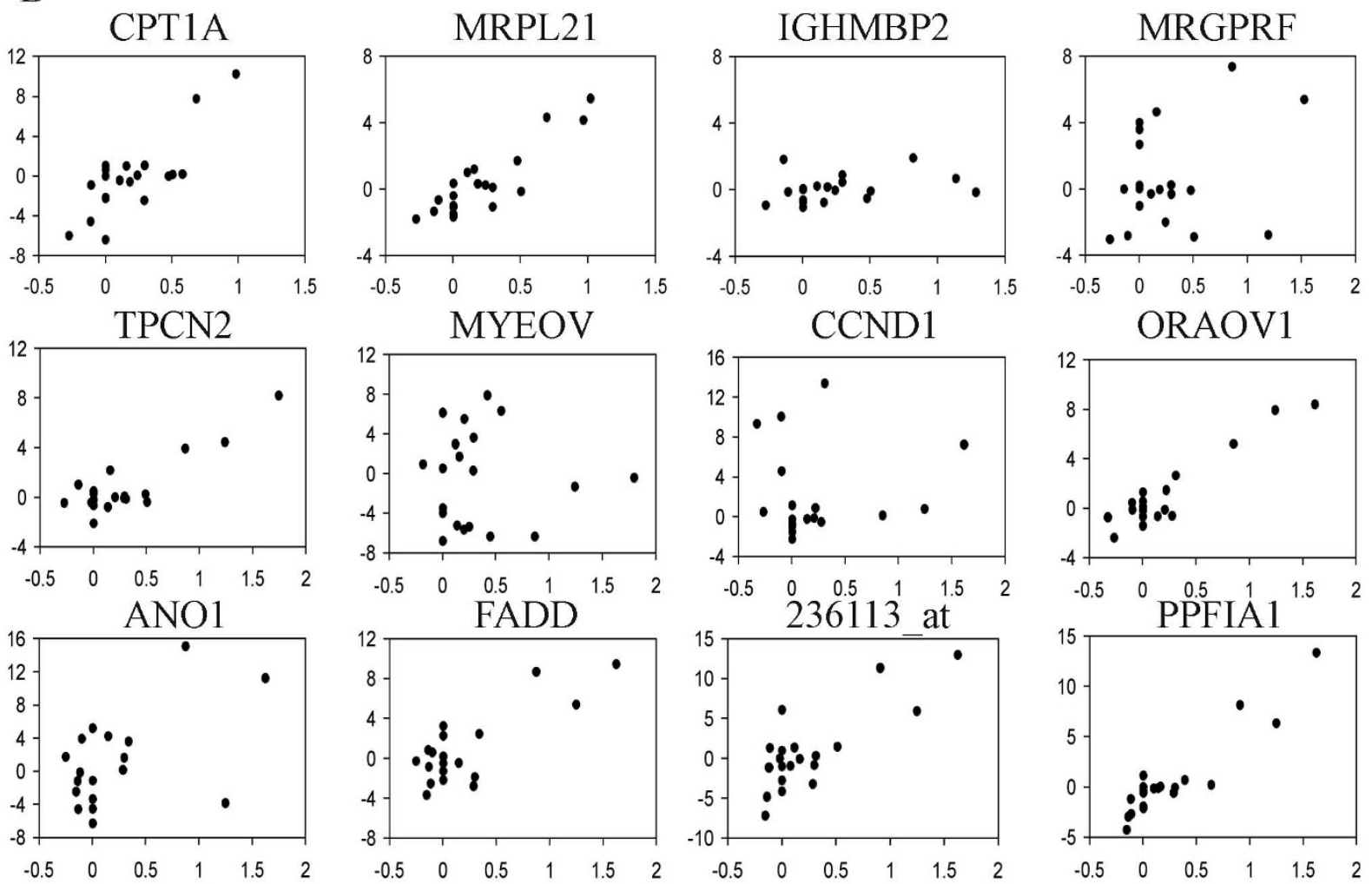

Figure 3 Correlation between DNA copy number and gene expression for genes in the high correlation region of chromosome 11q13.2q13.3. A. Locations of the genes and the unknown transcript in the region. B. DNA copy number ( $x$-axis, base-2 log-transformed ratio of cancer to normal) and gene expression (y-axis, base-2 log-transformed intensity) of each gene in the 20 lymph node metastatic OSCC. For genes measured by multiple probe sets, the probe set with the strongest correlation is shown.

tested a model using the first two PC scores of the 27 survival-associated transcripts. These two models were compared with AJCC stage alone, a current clinical guideline for survival prediction. The hazard ratios for five models with either stage or PC scores or with a combination of stage and PC scores are summarized in Table 2. Results from the ROC analysis for these five models are shown in Figure 4. The AUC for a model combining stage and the 122- or 27-transcript PC risk scores was signifi- cantly higher than for a model with stage alone $(p=0.044$ and 0.011 , respectively) (Figure $4 \mathrm{~A}$ and $4 \mathrm{~B}$ ).

\section{Discussion}

In this study, we analyzed the relationship between DNA copy number and gene expression in tumor cells from metastatic lymph nodes of 20 OSCC patients to: 1) identify the genes showing a significant correlation between DNA copy number and gene expression, and 2) deter- 
Table 1: Transcripts associated with survival

\begin{tabular}{|c|c|c|c|}
\hline Gene & Gene Title & By Probe Set & Note* \\
\hline C7orf30 & chromosome 7 open reading frame 30 & 226386_at & OSCC associated \\
\hline C8orf59 & chromosome 8 open reading frame 59 & 1555241_at & OSCC associated \\
\hline C8orf76 & chromosome 8 open reading frame 76 & 225702_at & \\
\hline CCT6A & chaperonin containing TCP1, subunit $6 \mathrm{~A}$ (zeta 1) & 201327_s_at & OSCC associated \\
\hline DCAF13 & DDB1 and CUL4 associated factor 13 & 225676_s_at & OSCC associated \\
\hline DNAJC8 & DnaJ (Hsp40) homolog, subfamily C, member 8 & 212490_at & OSCC associated \\
\hline FADD & Fas (TNFRSF6)-associated via death domain & 202535_at & OSCC associated \\
\hline FAF2 & Fas associated factor family member 2 & 212108_at & OSCC associated \\
\hline FUBP3 & far upstream element (FUSE) binding protein 3 & 212824_at & \\
\hline MRPL15 & mitochondrial ribosomal protein $\mathrm{L} 15$ & 218027_at & OSCC associated \\
\hline NFE2L2 & nuclear factor (erythroid-derived 2)-like 2 & 201146_at & OSCC associated \\
\hline OSMR & oncostatin $\mathrm{M}$ receptor & 1554008_at & OSCC associated \\
\hline PPFIA1 & $\begin{array}{l}\text { protein tyrosine phosphatase, receptor type, f polypeptide } \\
\text { (PTPRF), interacting protein (liprin), alpha } 1\end{array}$ & 210235_s_at; 202066_at; 210236_at & OSCC associated \\
\hline PURB & purine-rich element binding protein $B$ & 227718_at & OSCC associated \\
\hline RAD23B & RAD23 homolog B (S. cerevisiae) & 201223_s_at & \\
\hline RANBP6 & RAN binding protein 6 & 213019_at & \\
\hline SSSCA1 & Sjogren syndrome/scleroderma autoantigen 1 & 203114_at & OSCC associated \\
\hline STEAP3 & STEAP family member 3 & 218424_s_at; 1554830_a_at & OSCC associated \\
\hline TOR1B & torsin family 1 , member $B$ (torsin $B$ ) & 209593_s_at & OSCC associated \\
\hline TREM1 & triggering receptor expressed on myeloid cells 1 & 219434_at & OSCC associated \\
\hline UCK2 & uridine-cytidine kinase 2 & 209825_s_at & OSCC associated \\
\hline VPS54 & vacuolar protein sorting 54 homolog (S. cerevisiae) & 222627_at & OSCC associated \\
\hline YIPF5 & Yip1 domain family, member 5 & 224934_at & \\
\hline ZNF410 & zinc finger protein 410 & 209944_at & \\
\hline
\end{tabular}

*OSCC associated: gene is differentially expressed in OSCC vs. normal controls.

mine which if any copy number-associated genes from metastatic OSCC were associated with OSCC status and survival. We used the same cells to isolate and amplify DNA and RNA for high-throughput profiling whereas in previous studies, DNA and RNA were interrogated either from biopsy samples, or if tumor-cell specific, from two different laser microdissected cell populations. To our knowledge, this is the first time the genome and transcriptome of a solid tumor have been profiled and integrated using the genetic materials from the same cancer cell population enriched by LCM. This is also the first integrative analysis of DNA copy number and gene expression of tumor cells from metastatic OSCC lymph nodes.

There are a number of unresolved issues when interpreting data that integrate genome-wide DNA copy number and gene expression profiles. Study design, statistical analyses and quality of specimens are all factors that can all impact in how inferences are drawn between DNA copy number and gene expression. An early study in breast cancer indicated that at least $12 \%$ of all the variance in gene expression could be directly attributable to the underlying variation in DNA copy number [11]. As the authors pointed out, however, that was a significant underestimate as DNA was obtained from tissue biopsies with mixed tumor and stromal cells, reducing the ability to detect tumor-associated CNA. In a more recent study of non-small-cell lung carcinoma, $42 \%$ of the genes were classified as DNA copy number-driven [23]. However, the study did not enrich for tumor cells and dichotomized genome CNA as loss and gain only, thereby limiting the assessment of DNA copy number's impact on expression. Another breast cancer study that enriched the tumor cells via microdissection detected $46.7 \%$ of genes with a significant correlation between copy number and gene expression. However, that study did not appear to have adjusted for multiple comparisons and this may lead to over-estimation of the impact of copy number on gene expression 
Table 2: Hazard ratios association of AJCC stage and gene expression with survival

\begin{tabular}{|c|c|c|c|}
\hline \multirow[t]{2}{*}{ Model terms } & \multicolumn{3}{|c|}{ Hazard Ratio $\left(95 \% \mathrm{Cl}^{*}\right)$} \\
\hline & Unadjusted** & 122-gene PC + stage & 27-gene PC + stage \\
\hline AJCC stage & $2.24(1.35-3.71)$ & $2.13(1.29-3.54)$ & $2.04(1.24-3.35)$ \\
\hline 122-gene 1st PC $\mathrm{C}^{* * *}$ & $1.22(1.1-1.35)$ & $1.2(1.08-1.34)$ & \\
\hline 122-gene 2nd PC & $0.92(0.83-1.03)$ & $0.94(0.84-1.04)$ & \\
\hline 27-gene 1st PC & $1.28(1.14-1.44)$ & & $1.24(1.11-1.39)$ \\
\hline 27-gene 2nd PC & $1.34(1.07-1.69)$ & & $1.27(1.01-1.6)$ \\
\hline
\end{tabular}

[24]. Regardless of these limitations, data from these studies and ours does point that, in general increased DNA copy number was associated with increased expression. A critical issue is how to distinguish those copy number-associated genes which are the true "drivers" of carcinogenesis from those without functional relevance. We believe that determining which copy number-associated transcripts are associated with clinical characteristics and survival could be one approach to prioritize these transcripts further.

One interesting finding is the complexity seen between DNA copy number and gene expression for individual CNA regions. In the same DNA region, it was not unusual to find a mix of both genes the expression of which did and did not show a correlation with CNA. For instance, six genes and an unknown transcript located in the high correlation 11q13.2-q13.3 region showed a strong positive correlation between DNA copy number and gene expression, whereas the expression of five other genes located in the same region can not be explained by their CNA. Previous studies have linked five of the six copy number-associated genes in the 11q13.2-q13.3 region to cancer progression, including: 1) the genome amplification and overexpression of ORAOV1 and PPFIA1 associated with aggressive phenotypes in OSCC cell lines $[20,25] ; 2)$ the expression of the cell apoptotic signal mediator $F A D D$ associated with nodal metastasis in non-small cell lung cancer [26] and poor survival in laryngeal carcinoma [27]; and 3) the expression of CPT1A and MRPL21 associated with the development of colon cancer and breast cancer, respectively $[28,29]$. Therefore, at least some associations between DNA copy number and gene expression can be shown to have clinical or pathogenetic relevance, as opposed to only representing a DNA dosage effect.

Many of the gene candidates identified in this study have mechanistic relevance in regards to how cancer cells migrate from the primary organ to distant sites and sur- vive through this process and in their new environment. For example, out of the 116 OSCC specific genes, at least 20 are involved in either cell cycle or cell death. Similarly, for the 24 genes associated with survival, 6 genes are involved in cell death and 3 genes are involved in cell migration. The expression changes of these genes, which our study suggests occurs as a result of copy number alterations, may enhance the viability and migration capability of the cancer cells, thus assisting in the progression and spread of the OSCC. Further investigation of these genes to confirm their roles in OSCC metastasis is warranted.

Although genes with CNA-associated expression were located on all chromosomal arms studied, several clusters of such genes were observed (e.g., in the 11q13 region as described above and other regions such as 7q22.1 and 9p24.1 (see Figure 2)). Likely, a single CNA event in these regions would cause the dysregulated expression of multiple genes. It is generally believed that recurrent genome CNA regions harbour genes that are essential to cancer progression, but many current functional studies focus on identifying the single "key driver gene" in the region of CNA responsible for cancer progression. In a recent study on liver cancer, however, the over-expression of both $C I A P 1$ and Yap, as a result of genome amplification at mouse chromosome $9 \mathrm{qA} 1$, cooperatively promoted tumorigenesis [16]. In another study of glioblastoma, chromosome 12q13.3-14.1 amplification caused overexpression of genes (CDK4 and CENTG1) and a microRNA $(h s a-m i R-26 a)$, all of which were demonstrated to contribute to the progression of the cancer [30]. Thus, CNA in these regions may be an efficient mechanism for cancer cells to obtain functional benefits from multiple genes (and even microRNAs) to achieve a specific aberrant capability. Likewise, amplification at 11q13 is one of the most frequently observed CNA events in almost all solid tumors [31]. This single CNA event would likely cause the over-expression of genes such as 


\section{A Survival ROC Curve at 2 Years}

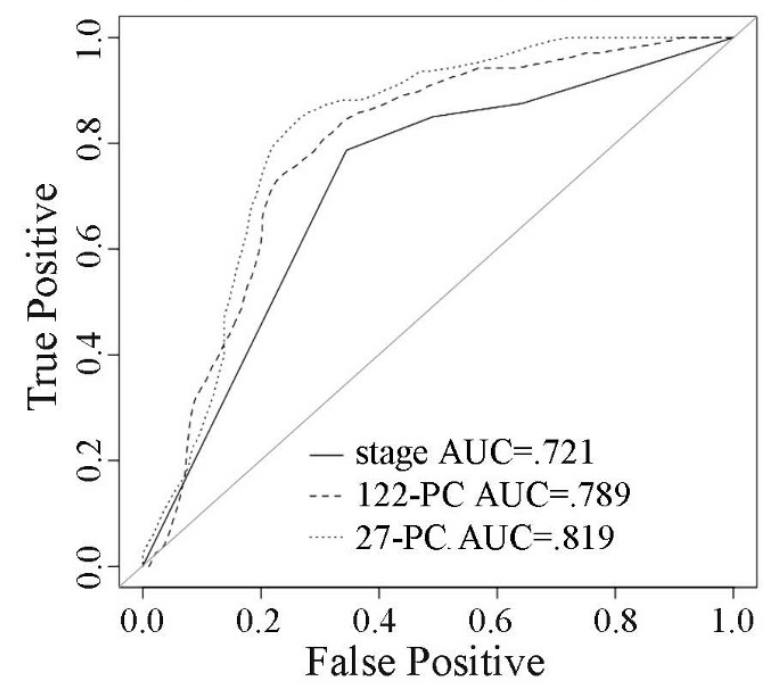

\section{B Survival ROC Curve at 2 Years}

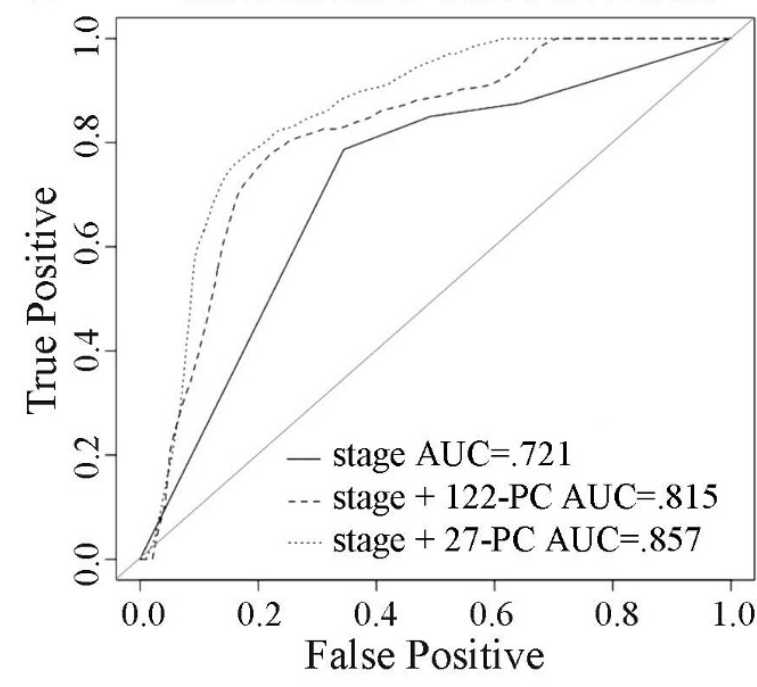

Figure 4 ROC analysis of 2-year survival comparing AJCC stage with gene expression data. $A, R O C$ curves for 2-year survival for models "stage," "122-PC," and "27-PC" B, ROC curves for 2-year survival for models "stage," "stage + 122-PC" and "stage + 27-PC".

ORAOV1, PPFIA1, and FADD, which, as mentioned above would synergize to promote OSCC progression. For this reason, genes with copy number associated expression in the regions of CNA reported in this study should be considered in a comprehensive way as concurrent dysregulation of these genes could contribute to the lymphotropic phenotype of these metastatic tumor cells.

The fact that among CNA-associated genes found in tumor cells isolated from nodal metastases, we found subsets that were significantly associated with OSCC status and survival using expression profiles of biopsy sam- ples from primary tumors which were not laser-dissected suggests that the signal from our copy number-associated genes was robust enough to overcome "noise" from the expression signal of bystander cells. Moreover, the fact that the two survival models incorporating the gene expression of the '122- or 27-transcript $\mathrm{PC}$ ' resulted in higher AUCs than a model with stage alone suggests that at least some of these copy number-associated transcripts may have prognostic relevance and could aid in explaining some of the outcome variation among tumors of the same stage. Thus, although there may be some genes irrelevant to the cancer process differentially expressed due to a dosage effect of CNA, our data demonstrate that integration of genome CNA with expression in tumor cells with an aggressive phenotype (i.e. lymphotropism) could uncover candidate biomarkers or therapeutic targets. However, larger scale integrative analysis with more patient tissue samples is needed for further validation and new discoveries of DNA copy number-associated genes. In addition, functional studies are warranted to assess the biological roles of these DNA copy number driven genes in the OSCC progression.

\section{Conclusions}

In this study, we have presented 1) an approach to systematically integrate DNA copy number aberrations and differential gene expression; 2) copy number-associated genes associated with OSCC status and survival; and 3) improved prognostication of 2-year survival using survival models based on copy number-associated transcripts compared to those using the current clinical standard, AJCC stage, alone. Further investigation is warranted to confirm these findings and examine the biologic role of these copy number-associated genes in OSCC metastasis and their potential as therapeutic targets.

\section{Methods}

\section{Tissue collection and specimen processing}

As described previously in Chen et al [21], we identified English-speaking patients 18 year of age or older with a first, primary OSCC at any of three University of Washington-affiliated hospitals: University of Washington Medical Center, Harborview Medical Center, and the Puget Sound Veterans Affairs Health Care System. Metastatic OSCC lymph nodes were snap-frozen at time of surgical resection and stored in liquid nitrogen until use. Tissue preparation, LCM of tumor cells, and extraction of DNA and RNA from the same LCM-harvested cell population was performed as previously described [32]. Purified RNA was stored in $-80^{\circ} \mathrm{C}$ until use. Quantity and quality of purified RNA and DNA from each specimen was determined by ND-1000 spectrophotometer (NanoDrop Technologies, Wilmington, DE) and Agilent 2100 bioanalyzer (Agilent Technologies, Santa Clara, CA).. 
Purified DNA was dried on a Savant DNA Speed Vac 110 (Global Medical Instrumentation, Ramsey, MN), re-dissolved as $50 \mathrm{ng} / \mu \mathrm{l}$ in $10 \mathrm{mM}$ Tris- $\mathrm{HCl} \mathrm{pH} 8$ with $0.1 \mathrm{mM}$ EDTA, and stored at $-20^{\circ} \mathrm{C}$ until use [32].

\section{Genome profiling with SNP array}

The DNA was hybridized onto Affymetrix Human Mapping 250 K Nsp I SNP array (Affymetrix Inc., Santa Clara, CA) following the manufacturer's instructions. Briefly, $250 \mathrm{ng}$ of purified genomic DNA was digested with restriction enzyme Nsp I, ligated to an Nsp I adaptor, and PCR amplified. After purification, $90 \mu \mathrm{g}$ of amplified DNA were fragmented, biotin-labeled, and hybridized to the SNP array. Intensity of the hybridized feature was acquired with Affymetrix GCOS (v1.4) software. SNP genotyping was performed with the Robust Linear Model with Mahalanobis distance classifier algorithm (BRLMM) in Affymetrix Genotyping Console (v3.0) software. Quality of the genome profiling process was assessed by the yield and length range of the amplified DNA, the length range of the fragmented DNA, and the SNP call rate following the manufacturer's instructions [32]. The SNP array results were normalized through median smoothing coupled with the Invariant Set Normalization method in dChip [33]. We took the log2 ratio between our normalized SNP array results and 2 which is the expected number of DNA copies in a normal cell and estimated the DNA copy number using the $R$ package cghFlasso in reference to the Affymetrix 48 HapMap normal samples. cghFlasso infers the underlying piecewise linear DNA copy number profiles with fused lasso regression, and calls gains and losses by controlling the overall false discovery rate [34]. The location of each SNP probe set was mapped to the human genome (Build 36.1) using Affymetrix annotation file 'Mapping250K_Nsp.na26.annot.csv'. The genome DNA copy number of a transcript was calculated as: 1) the average genome DNA copy number inferred from all the SNPs within the transcript if the transcript overlapped with 5 or more SNPs; 2) the average of the genome DNA copy numbers inferred by the closest 5 SNPs within a $250 \mathrm{~kb}$ neighboring region of the transcript; or 3) the average of the genome DNA copy numbers inferred by all the SNPs within $250 \mathrm{~kb}$ of the transcript if there were fewer than 5 SNPs in this region.

\section{Transcriptome profiling}

The RNA was hybridized onto Affymetrix Human Genome U133 Plus 2.0 array (Affymetrix Inc., Santa Clara, CA) as previously described [7]. Briefly, $40 \mathrm{ng}$ of purified total RNA was amplified using Arcturus RiboAmp Plus 1.5-round kit (Molecular Devices) following the manufacturer's instructions. Amplified RNA was biotin-labeled and fragmented using Affymetrix GeneChip IVT Labeling kit and hybridized to the U133 Plus 2.0 array following manufacturer's protocol. Intensity of the hybridized feature was acquired with Affymetrix GCOS (v1.4) software. Quality of the transcriptome profiling process was assessed by the yield and length range of the amplified RNA, the length range of the fragmented RNA, the average background, the "Present" call rate, and the 3'/5' ratio for glyceraldehyde-3-phosphate dehydrogenase and $ß$-actin following the manufacturer's instructions [32]. Gene expression profiles were normalized using the $\mathrm{R}$ package gcRMA http://www.bioconductor.org.The location of each transcript measured by each probe set was mapped to the human genome (Build 36.1) using Affymetrix annotation file 'HG-U133_Plus_2.na27'.

\section{Transcript filtering}

We eliminated transcripts which: 1) had no alignment information (e.g., control probes) or no unique alignment to the human genome; 2) were on the sex chromosome and mitochondrial DNA; 3) had no expression values greater than 3 on a $\log 2$ scale in at least 3 samples; and 4) had an inter-quartile range of expression less than 0.1 on a $\log 2$ scale [21].

\section{Analysis of the association between DNA copy number and gene expression}

To ensure that the median of the expression data from any sample or transcript was around zero with the same variation across samples or transcripts, a second normalization was employed after transcript filtering using the following formula:

$$
\hat{\mathrm{Y}}=(\mathrm{Yi}-\tilde{\mathrm{Y}}) / \operatorname{median}(|\mathrm{Yi}-\tilde{\mathrm{Y}}|)(\mathrm{i}=1,2, \ldots, \mathrm{n})
$$

where ? is the median of Ys and $|\mathrm{Y}|$ is the absolute value of Y. Our goal was to find genes with expression associated with DNA copy number. For this purpose, we employed robust regression to evaluate the correlation between gene expression and DNA copy number across OSCC samples. Compared to least square regression (LSR), robust regression analysis provides an alternative when fundamental assumptions are unfulfilled by the nature of the data. In our case, it was equality of the error variance along the predicted line that did not hold. We used rlm function from $\mathrm{R}$ package MASS, in which iteratively reweighted least square algorithm was employed to estimate parameters in the regression model. For each gene, we fitted the model:

$$
\mathrm{Yi}=\alpha+\beta \mathrm{Xi}(\mathrm{i} \text { is } 1,2, \ldots, \text { number of transcripts })
$$

where $\mathrm{Yi}$ was the gene expression and $\mathrm{Xi}$ was the DNA copy number estimated from the SNPs. Two-sided Pvalue for the test of null hypothesis $\beta=0$ was calculated using the t-statistics from the robust regression with a 
degree of freedom $(\mathrm{df})=\mathrm{N}-2$ where $\mathrm{N}$ is the number of samples.

\section{Enrichment analysis of DNA copy number-associated transcripts}

We identified copy number-associated transcripts meeting a False Discovery Rate (FDR) of $<1 \%$ ( $n=530$ transcripts) to determine if these represented an enriched subset of transcripts associated with OSCC status or survival by using our previously published, independent dataset comprising of 167 OSCC primary tumors and 45 normal controls [21], from which we excluded 19 patients whose metastatic lymph nodes were used for generating the CNA-associated transcripts. (1 OSCC patient whose metastatic lymph nodes were used to identify CNA associated transcripts was not part of Chen et al. 2008.). The association between the expression of these transcripts and being a case in this independent dataset comprising of 148 OSCC cases and 45 controls was assessed using a linear regression implemented by GenePlus software $[35,36]$. To determine the association between expression and survival, we analyzed expression array results for the subset of the 148 OSCC patients for which we had at least four months of follow-up time to reduce the inclusion of deaths from patients due to co-morbidities rather than tumor biology $(\mathrm{n}=132)$. The association between expression and survival was estimated using Cox-regression adjusting for age and sex. A dummy variable was created for sex. Then to determine whether our copy numberassociated transcripts $(n=530)$ represented an enriched subset of transcripts associated with OSCC (vs. normal control), we compared the observed Z-score distribution of the 530 transcripts to the expected Z-score distribution from randomly selecting a subset of transcripts the same size one thousand times. This comparison of Zscore distributions was repeated for survival.

\section{Comparing survival prediction models with AJCC stage}

Using the subset of copy number-associated transcripts which were found to be associated with either the OSCC status or survival in our enrichment analysis above, we determined whether a model which incorporates gene expression data predicts survival better than stage alone. We first reduced the dimensionality of the gene expression data by calculating the first two principal components summarizing the expression of a subset of copy number-associated transcripts which in our enrichment analysis were found to be either differentially expressed in OSCC vs. normal control $(\mathrm{n}=122)$ or associated with survival $(\mathrm{n}=27)$ using Matlab version R2006b. We decided to include the transcripts associated with OSCC (vs. normal control) in the survival prediction based on our previously reported finding that a group of 131 genes differentially expressed genes in OSCC identified OSCC patients at high risk for poor survival [22]. We fit five summary Cox models of OSCC-specific survival. The first model contained stage alone as a continuous predictor, the second contained the first and second principal components summarizing expression of the 122 transcripts, and the third model contained first and second principal components for the 27 transcripts. The fourth and fifth models included principal components for the 122 and 27 transcripts respectively along with stage. Risk scores were calculated using a jackknife leave-one-out analysis for 4 models: two models using the first two principal component scores from the two subsets of correlated transcripts ('122 $\mathrm{PC}$ ' and '27 PC'); and two other models combining AJCC stage and the principal component scores from either of the two transcript subsets ('stage $+122 \mathrm{PC}$ ' and 'stage $+27 \mathrm{PC}$ '). For each model and for AJCC stage, we then used an adapted Receiver Operating Characteristics (ROC) analysis [37] to construct ROC curves for predicting two year survival as previously described [22].

\section{Ingenuity Pathways Analysis}

The lists of the 122 and 27 transcripts were analyzed using Ingenuity Pathway Analysis software (IPA, version 8.5, Ingenuity Systems, http://www.ingenuity.com Core Analysis. We used the default settings so the Functions/ Pathways/Tox list Analyses were performed with "Ingenuity Knowledge Base (Genes Only)" as reference set and both direct and indirect relationships were considered in the Network Analysis.

\section{Additional material}

Additional file $\mathbf{1}$ Table S1. Age, gender, cancer site and stage of OSCC
patients used to identify copy number associated gene-expression
Additional file $\mathbf{2}$ Figure $\mathbf{S 1}$. Consensus plot of genome-wide copy num-
ber gains and losses in the 20 lymph node metastatic OSCC
Additional file $\mathbf{3}$ Table S2. Transcripts* and the SNPs in their neighboring
regions
Additional file $\mathbf{4}$ Table S3. Transcripts differentially expressed in OSCC vs.
controls

\section{Competing interests}

The authors declare that they have no competing interests.

\section{Authors' contributions}

CX carried out sample processing; laser-capture microdissection; genome profiling; transcriptome profiling; Ingenuity Pathway Analysis; participated in data analysis design; and drafted the manuscript. YL carried out statistical analysis of estimating DNA copy number and its association with gene expression; identified associations between copy number-associated genes and OSCC status and clinical outcomes; and revised; and revised the manuscript. PW gave guidance on statistical methodologies and tools; provided insightful explanation of the analytical results and major revisions to the manuscript. WF carried out the gene expression analysis; principal component analysis; and participated in data analysis design and revision of the manuscript. TCR carried out survival prediction modelling and participated in revision of the manuscript. MPU provided pathological support and participated in revision of the manuscript. JRH performed sample management and sample processing; and participated in revision of the manuscript. PL carried out the extraction of clinical information 
and participated in revision of the manuscript. DRD prepared datasets and conducted statistical analyses of clinical and outcomes data. NDF carried out patient recruitment and sample collection; and participated in overall design. LPZ participated in overall design and provided statistical guidance to WF for analysis of the gene expression data. SMS provided epidemiological support, guidance to DRD, and participated in overall design, planning of the data analysis; and provided major revisions to the manuscript. CC, as the principal investigator of the parent study ( $\mathrm{NIH}$ RO1CA095419-06A1), provided the biospecimens and study participants-associated relevant data that were obtained through in-person baseline and follow-up interviews and medical record abstraction; provided guidance to PL in medical chart abstraction of clinical information; provided guidance to JRH to carry out sample management and processing; participated in overall study design and data analysis design; and provided major revisions to the manuscript. EM carried out overall study design; coordination of collaborations; protocol development; sample processing; experimental design; transcriptome profiling; Ingenuity Pathway Analysis; and data analysis design; provided guidance to CX to execute study design and to PL in medical chart abstraction for clinical information; and drafted and provided major revisions to the manuscript. All authors read and approved the final manuscript.

\section{Acknowledgements}

This work was supported in part by grants 5KL2RR025015-03 from National Center for Research Resources, National Institutes of Health (NIH); Amos Medical Faculty Development Program Award from The Robert Wood Johnson Foundation; Early Physician-Scientist Career Development Award from the Howard Hughes Medical Institute; R01CA095419 from the National Cancer Institute, NIH; Small Grants Translational Research Projects Award from the Institute of Translational Health Sciences, University of Washington supported by grant UL1RR025014 from the National Center for Research Resources, NIH; center funds from the Department of Otolaryngology - Head and Neck Surgery, University of Washington; and by resources from and use of facilities at the VA Puget Sound Health Care System, Fred Hutchinson Cancer Research Center, University of Washington Medical Center and Harborview Medical Center, Seattle, Washington.

\section{Author Details}

'Department of Otolaryngology-Head and Neck Surgery, University of Washington, Seattle, WA 98195, USA, 2Program in Biostatistics \& Biomathematics, Public Health Sciences Division, Fred Hutchinson Cancer Research Center, Seattle, WA 98109, USA, 3Program in Cancer Prevention and Biostatistics, Public Health Sciences Division, Fred Hutchinson Cancer Research Center, Seattle, WA 98109, USA, ${ }^{4}$ Department of Biostatistics, University of Washington, Seattle, WA 98195, USA, ${ }^{5}$ Department of Pathology, University of Washington, Seattle, WA 98195, USA, ${ }^{6}$ Program in Epidemiology, Public Health Sciences Division, Fred Hutchinson Cancer Research Center, Seattle, WA 98109, USA, ${ }^{7}$ Department of Epidemiology, University of Washington, Seattle, WA 98195, USA, ${ }^{8}$ Clinical Research Division, Fred Hutchinson Cancer Research Center, Seattle, WA 98109, USA and ${ }^{9}$ Surgery and Perioperative Care Service, VA Puget Sound Health Care System, Seattle, Washington 98108, USA

Received: 10 February 2010 Accepted: 11 June 2010

Published: 11 June 2010

\section{References}

1. Liu CJ, Liu TY, Kuo LT, Cheng HW, Chu TH, Chang KW, Lin SC: Differential gene expression signature between primary and metastatic head and neck squamous cell carcinoma. J Pathol 2008, 214:489-497.

2. Garzino-Demo P, Dell'Acqua A, Dalmasso P, Fasolis M, La Terra Maggiore GM, Ramieri G, Berrone S, Rampino M, Schena M: Clinicopathological parameters and outcome of 245 patients operated for oral squamous cell carcinoma. J Craniomaxillofac Surg 2006, 34:344-350.

3. Imre K, Pinar E, Oncel S, Calli C, Tatar B: Predictors of extracapsular spread in lymph node metastasis. Eur Arch Otorhinolaryngol. 2008, 265:337-339.

4. Puri SK, Fan CY, Hanna E: Significance of extracapsular lymph node metastases in patients with head and neck squamous cell carcinoma. Current Opinion in Otolaryngology \& Head \& Neck Surgery 2003, 11:119-123.

5. Schmalbach CE, Chepeha DB, Giordano TJ, Rubin MA, Teknos TN, Bradford CR, Wolf GT, Kuick R, Misek DE, Trask DK, et al:: Molecular profiling and the identification of genes associated with metastatic oral cavity/pharynx squamous cell carcinoma. Arch Otolaryngol Head Neck Surg 2004 130:295-302.

6. Roepman P, de JA, Groot Koerkamp MJ, Kummer JA, Slootweg PJ, Holstege FC: Maintenance of Head and Neck Tumor Gene Expression Profiles upon Lymph Node Metastasis. Cancer Res 2006, 66:11110-11114.

7. Mendez E, Fan W, Choi P, Agoff SN, Whipple M, Farwell DG, Futran ND, Weymuller EA Jr, Zhao LP, Chen C: Tumor-specific genetic expression profiles of metastatic oral squamous cell carcinoma. Head Neck 2007, 29:803-814

8. Albertson DG, Collins C, Mccormick F, Gray JW: Chromosome aberrations in solid tumors. Nat Genet 2003, 34:369-376.

9. Lastowska M, Viprey V, Santibanez-Koref M, Wappler I, Peters H, Cullinane C, Roberts P, Hall AG, Tweddle DA, Pearson AD, et al:: Identification of candidate genes involved in neuroblastoma progression by combining genomic and expression microarrays with survival data. Oncogene 2007, 26:7432-7444

10. Kotliarov Y, Kotliarova S, Charong N, Li A, Walling J, Aquilanti E, Ahn S, Steed ME, Su Q, Center A, et al:: Correlation analysis between singlenucleotide polymorphism and expression arrays in gliomas identifies potentially relevant target genes. Cancer Res 2009, 69:1596-1603.

11. Pollack JR, Sorlie T, Perou CM, Rees CA, Jeffrey SS, Lonning PE, Tibshirani $R$, Botstein D, Borresen-Dale AL, Brown PO: Microarray analysis reveals a major direct role of DNA copy number alteration in the transcriptional program of human breast tumors. Proc Natl Acad Sci USA 2002, 99:12963-12968.

12. Boehm JS, Zhao JJ, Yao J, Kim SY, Firestein R, Dunn IF, Sjostrom SK, Garraway LA, Weremowicz S, Richardson AL, et al:: Integrative genomic approaches identify IKBKE as a breast cancer oncogene. Cell 2007, 129:1065-1079.

13. Adelaide J, Finetti P, Bekhouche I, Repellini L, Geneix J, Sircoulomb F, Charafe-Jauffret E, Cervera N, Desplans J, Parzy D, et al: Integrated profiling of basal and luminal breast cancers. Cancer Res 2007 67:11565-11575.

14. Vincent-Salomon A, Lucchesi C, Gruel N, Raynal V, Pierron G, Goudefroye R, Reyal F, Radvanyi F, Salmon R, Thiery JP, et al: Integrated genomic and transcriptomic analysis of ductal carcinoma in situ of the breast. Clin Cancer Res 2008, 14:1956-1965.

15. Etemadmoghadam D, deFazio A, Beroukhim R, Mermel C, George J, Getz G, Tothill R, Okamoto A, Raeder MB, Harnett P, et al:: Integrated genomewide DNA copy number and expression analysis identifies distinct mechanisms of primary chemoresistance in ovarian carcinomas. Clin Cancer Res 2009, 15:1417-1427.

16. Zender L, Spector MS, Xue W, Flemming P, Cordon-Cardo C, Silke J, Fan ST, Luk JM, Wigler M, Hannon GJ, et al: Identification and validation of oncogenes in liver cancer using an integrative oncogenomic approach. Cell 2006, 125:1253-1267.

17. Walker BA, Leone PE, Jenner MW, Li C, Gonzalez D, Johnson DC, Ross FM Davies FE, Morgan GJ: Integration of global SNP-based mapping and expression arrays reveals key regions, mechanisms, and genes important in the pathogenesis of multiple myeloma. Blood 2006, 108:1733-1743.

18. Andersen CL, Wiuf C, Kruhoffer M, Korsgaard M, Laurberg S, Orntoft TF: Frequent occurrence of uniparental disomy in colorectal cancer. Carcinogenesis 2007, 28:38-48.

19. Chen Y, Chen C: DNA copy number variation and loss of heterozygosity in relation to recurrence of and survival from head and neck squamous cell carcinoma. Head Neck 2008, 30:1361-1383.

20. Xia J, Chen Q, Li B, Zeng X: Amplifications of TAOS1 and EMS1 genes in oral carcinogenesis: association with clinicopathological features. Oral Oncology 2007, 43:508-514.

21. Chen C, Mendez E, Houck J, Fan W, Lohavanichbutr P, Doody D, Yueh B, Futran ND, Upton M, Farwell DG, et al: Gene expression profiling identifies genes predictive of oral squamous cell carcinoma. Cancer Epidemiol Biomarkers Prev 2008, 17:2152-2162.

22. Mendez E, Houck JR, Doody DR, Fan W, Lohavanichbutr P, Rue TC, Yueh B, Futran ND, Upton MP, Farwell DG, et al:: A genetic expression profile associated with oral cancer identifies a group of patients at high risk of poor survival. Clin Cancer Res 2009, 15:1353-1361.

23. Broet P, Camilleri-Broet S, Zhang S, Alifano M, Bangarusamy D, Battistella M, Wu Y, Tuefferd M, Regnard JF, Lim E, et al:: Prediction of clinical outcome in multiple lung cancer cohorts by integrative genomics: 
implications for chemotherapy selection. Cancer Res 2009, 69:1055-1062.

24. Natrajan R, Weigelt B, Mackay A, Geyer FC, Grigoriadis A, Tan DS, Jones C, Lord CJ, Vatcheva R, Rodriguez-Pinilla SM, et al: An integrative genomic and transcriptomic analysis reveals molecular pathways and networks regulated by copy number aberrations in basal-like, HER2 and luminal cancers. Breast Cancer Res Treat 2009, 121(3):575-89.

25. Tan KD, Zhu Y, Tan HK, Rajasegaran V, Aggarwal A, Wu J, Wu HY, Hwang J, Lim DT, Soo KC, et al:: Amplification and overexpression of PPFIA1, a putative 11q13 invasion suppressor gene, in head and neck squamous cell carcinoma. Genes Chromosom Cancer 2008, 47:353-362.

26. Shin MS, Kim HS, Lee SH, Lee JW, Song YH, Kim YS, Park WS, Kim SY, Lee SN, Park JY, et al:: Alterations of Fas-pathway genes associated with nodal metastasis in non-small cell lung cancer. Oncogene 2002, 21:4129-4136

27. Gibcus JH, Menkema L, Mastik MF, Hermsen MA, de Bock GH, Van Velthuysen ML, Takes RP, Kok K, Alvarez Marcos CA, Van Der Laan BF, et al: Amplicon mapping and expression profiling identify the Fasassociated death domain gene as a new driver in the 11q13.3 amplicon in laryngeal/pharyngeal cancer. Clin Cancer Res 2008, 13:6257-6266.

28. Mazzarelli P, Pucci S, Bonanno E, Sesti F, Calvani M, Spagnoli LG: Carnitine palmitoyltransferase I in human carcinomas: a novel role in histone deacetylation? Cancer Biology \& Therapy 2007, 6:1606-1613.

29. Kim JC, Kim SY, Roh SA, Cho DH, Kim DD, Kim JH, Kim YS: Gene expression profiling: canonical molecular changes and clinicopathological features in sporadic colorectal cancers. World Journal of Gastroenterology 2008, 14:6662-6672.

30. Kim H, Huang W, Jiang X, Pennicooke B, Park PJ, Johnson MD: Integrative genome analysis reveals an oncomir/oncogene cluster regulating glioblastoma survivorship. Proc Natl Acad Sci USA 2010, 107:2183-2188.

31. Beroukhim R, Mermel CH, Porter D, Wei G, Raychaudhuri S, Donovan J, Barretina J, Boehm JS, Dobson J, Urashima M, et al.: The landscape of somatic copy-number alteration across human cancers. Nature 2010, 463:899-905.

32. Xu C, Houck JR, Fan W, Wang P, Chen Y, Upton M, Futran ND, Schwartz SM, Zhao LP, Chen C, et al.: Simultaneous isolation of DNA and RNA from the same cell population obtained by laser capture microdissection for genome and transcriptome profiling. J Mol Diagn 2008, 10:129-134.

33. Lin M, Wei LJ, Sellers WR, Lieberfarb M, Wong WH, Li C: dChipSNP. significance curve and clustering of SNP-array-based loss-ofheterozygosity data. Bioinformatics 2004, 20:1233-1240.

34. Tibshirani R, Wang P: Spatial smoothing and hot spot detection for $\mathrm{CGH}$ data using the fused lasso. Biostatistics 2008, 9:18-29.

35. Zhao LP, Prentice R, Breeden L: Statistical modeling of large microarray data sets to identify stimulus-response profiles. Proc Natl Acad Sci USA 2001, 98:5631-5636

36. Thomas JG, Olson JM, Tapscott SJ, Zhao LP: An efficient and robust statistical modeling approach to discover differentially expressed genes using genomic expression profiles. Genome Res 2001 11:1227-1236

37. Heagerty PJ, Lumley T, Pepe MS: Time-dependent ROC curves for censored survival data and a diagnostic marker. Biometrics 2000, 56:337-344.

doi: $10.1186 / 1476-4598-9-143$

Cite this article as: Xu et al., Integrative analysis of DNA copy number and gene expression in metastatic oral squamous cell carcinoma identifies genes associated with poor survival Molecular Cancer 2010, 9:143

\section{Submit your next manuscript to BioMed Central} and take full advantage of:

- Convenient online submission

- Thorough peer review

- No space constraints or color figure charges

- Immediate publication on acceptance

- Inclusion in PubMed, CAS, Scopus and Google Scholar

- Research which is freely available for redistribution

Submit your manuscript at www.biomedcentral.com/submit
C BioMed Central 